\title{
Associação entre teor de nitrogênio em cultivares de arroz e ataque de Oryzophagus oryzae (Costa Lima) (Coleoptera: Curculionidae)
}

\author{
Association between nitrogen content in rice cultivars and the damage of Oryzophagus oryzae \\ (Costa Lima) (Coleoptera: Curculionidae)
}

\author{
Uemerson Silva da Cunha ${ }^{1}$ Jairo João Carbonari ${ }^{2}$ José Djair Vendramim $^{3}$ \\ José Francisco da Silva Martins ${ }^{4}$
}

\section{RESUMO}

O gorgulho-aquático-do-arroz Oryzophagus oryzae (Costa Lima) (Coleoptera: Curculionidae) é um dos insetos mais prejudiciais à cultura do arroz irrigado, devido aos danos causados pelas suas larvas às raízes da planta. A associação entre o teor de nitrogênio e o ataque de $\boldsymbol{O}$. oryzae foi avaliada nas cultivares "BR IRGA 410", "BRS Firmeza", "Dawn" e "BRS Atalanta", diferenciadas pelo ciclo de desenvolvimento fenológico e pelo grau de resistência ao inseto, em experimentos no campo, com e sem chance de escolha de hospedeiro, na Embrapa Clima Temperado, Pelotas, Rio Grande do Sul. Determinaram-se o número de folhas lesionadas, o número e o peso de larvas do inseto e, em três épocas distintas, o teor de nitrogênio na parte aérea e nas raízes das plantas. A alimentação dos adultos de $\boldsymbol{O}$. oryzae foi estimulada pelo teor de nitrogênio da parte aérea das plantas. A cultivar "Dawn", que apresentou menor número de larvas $e$ maior ciclo de desenvolvimento fenológico, foi a única a recuperar o teor de nitrogênio. $O$ dano às raízes, causado por larvas de $\boldsymbol{O}$. oryzae, reduziu o teor de nitrogênio e, conseqüentemente, provocou perda na produção de grãos.

Palavras-chave: Oryza sativa, gorgulho-aquático, resistência de plantas, nutriente.

\section{ABSTRACT}

The rice water weevil Oryzophagus oryzae (Costa Lima) (Coleoptera: Curculionidae) is one of the most important pests in irrigated rice culture due to the damage caused by the larvae in the roots. The association between the nitrogen content and the $O$. oryzae damage was evaluated on BR IRGA 410, BRS Firmeza, Dawn and BRS Atalanta cultivars differentiated by the phenological development cycle and the insect resistance

\begin{abstract}
degree. The experiments were conducted under field conditions, with and without chance of host plant choice, at Embrapa Clima Temperado, Pelotas, RS. The following parameters were evaluated: number of damaged leaves, number and weight of larvae. The nitrogen content in the leaves and roots were evaluated at three different rice growth stages. The adult feeding of $\boldsymbol{O}$. oryzae were stimulated by the content of nitrogen in the foliage of the plant. The cultivar Dawn was the only one to revover the nitrogen content, due to the presence of the smallest number of larvae and the largest development cycle in compare to other cultivars. The root damage caused by $\boldsymbol{O}$. oryzae larvae decreased the nitrogen content, and consequently reduced the rice yield.
\end{abstract}

Key words: Oryza sativa, rice water weevil, plant resistance, nutrient.

\section{INTRODUÇÃO}

A lavoura orizícola irrigada do Brasil é danificada por diversas espécies de insetos, destacando-se Oryzophagus oryzae (Costa Lima) (Coleoptera: Curculionidae), pelos consideráveis danos causados às plantas (FERREIRA \& MARTINS, 1984; MARTINS et al., 2004a; b). Na fase adulta, $\boldsymbol{O}$. oryzae alimenta-se de folhas e oviposita nas partes submersas das plantas de arroz, enquanto as larvas, ao alimentarem-se do sistema radicular, afetam o crescimento e o desenvolvimento das plantas, em lavouras irrigadas (MARTINS et al., 2004a).

'Departamento de Fitossanidade, Faculdade de Agronomia "Eliseu Maciel” (FAEM), Universidade Federal de Pelotas (UFPel), CP 354, 96010-900, Pelotas, RS, Brasil. E-mail: uscunha@ yahoo.com.br. Autor para correspondência.

${ }^{2}$ Ministério da Agricultura, Pecuária e Abastecimento, Serviço de Defesa Vegetal. Av. Loureiro da Silva, 515, sala 509, 96010-420, Porto Alegre, RS, Brasil.

${ }^{3}$ Departamento de Entomologia, Fitopatologia e Zoologia Agrícola, Escola Superior de Agricultura Luiz de Queiroz (ESALQ), Universidade de São Paulo (USP), CP 9, 13418-900, Piracicaba, SP, Brasil.

${ }^{4}$ Embrapa Arroz e Feijão, CP 179, 75375-000, Santo Antônio de Goiás, GO, Brasil. 
No sistema de cultivo, $\boldsymbol{O}$. oryzae atacava apenas áreas localizadas da lavoura, passando a ocorrer de forma generalizada, em conseqüência das constantes modificações tecnológicas nos diferentes sistemas (MARTINS \& FERREIRA, 1980; MARTINS \& BOTTON, 1996).

Aproximadamente $25 \%$ da área de arroz cultivada no Rio Grande do Sul é infestada por $\boldsymbol{O}$. oryzae, causando perdas anuais de produção de 10 a 30\% (MARTINS, 1990; MARTINS et al., 2004a). Mesmo utilizando práticas culturais como limpeza dos canais de irrigação, eliminação de restos de cultura e aplainamento do solo para a redução da população do inseto (MARTINS \& BOTTON, 1996), ainda predomina a aplicação de inseticidas (MARTINS et al., 1996; MARTINS et al., 2004a).

Resultados positivos obtidos com $\boldsymbol{O}$. oryzae, no Brasil (MARTINS \& TERRES, 1995), e com Lissorhoptrus oryzophilus Kuschel (Coleoptera: Curculionidae), nos EUA (SMITH \& ROBINSON, 1982; N'GUESSAN et al., 1994), indicam que o uso de cultivares resistentes são alternativas promissoras para o controle desses insetos, o que implica a redução de custos operacionais, a diminuição de riscos de contaminação do ambiente e, conseqüentemente, o aumento da sustentabilidade do sistema produtivo. Resistência dos tipos antixenose, antibiose e tolerância a gorgulhos-aquáticos têm sido encontradas em plantas de arroz (BOWLING, 1980). A tolerância, através de baixos índices de redução de produção, mesmo sob elevado número de larvas, pode ter como causa importante a habilidade de alguns cultivares em absorver determinados nutrientes, como o nitrogênio, em maior quantidade que outros sob as mesmas condições. $\mathrm{O}$ efeito da adubação nitrogenada, na recuperação de raízes atacadas por larvas de $\boldsymbol{O}$. oryzae, mostra que a capacidade de recuperação do sistema radicular de plantas da cultivar "Taim" foi maior que na "Chuî", possivelmente devido à diferença de duração do ciclo entre as cultivares, médio e precoce, respectivamente (CUNHA et al., 2001). Além disso, o nitrogênio exerce efeito diferenciado sobre adultos e larvas de gorgulhos-aquáticos, dependendo da fonte do nutriente, da época de aplicação e da concentração nas plantas (BANG \& TUGWELL, 1976; STOUT et al., 1998).

O consumo de folhas por L. oryzophilus aumenta, proporcionalmente, com o incremento de nitrogênio total na planta (BANG \& TUGWELL, 1976), onde as doses crescentes do nutriente, aplicadas em plantas de arroz antes da irrigação por inundação, foram associadas com o aumento da população larval desse inseto (BOWLING, 1963; STOUT et al., 1998) e de Lissorhoptrus brevirostris (Suffrían) (MENESES \&
ELIZALDE, 1980). A aplicação de nitrogênio na forma de sulfato de amônia reduz temporariamente a população larval do inseto, proporcionando maior recuperação do sistema radicular danificado (MARTINS et al., 1986). No entanto, a aplicação, na forma de uréia, em cobertura na água de irrigação, não ocasionou mortalidade de larvas de O. oryzae (OLIVEIRA, 1980; CUNHA et al., 2001), contrariando hipótese de efeito inseticida do fertilizante. Com base no exposto, torna-se importante avaliar a relação existente entre o teor de nitrogênio e a resistência de cultivares de arroz irrigado a $\boldsymbol{O}$. oryzae.

O objetivou deste trabalho foi relacionar o teor de nitrogênio na parte aérea e nas raízes de plantas com o ataque de $\boldsymbol{O}$. oryzae, em quatro cultivares de arroz irrigado, diferenciadas pelo grau de resistência ao inseto e pelo ciclo fenológico de cada cultivar.

\section{MATERIAL E MÉTODOS}

A associação entre a população de $\boldsymbol{O}$. oryzae e o teor de nitrogênio foi avaliada nas cultivares "BR IRGA 410" e "BRS Atalanta", consideradas resistentes ao inseto e com ciclo de desenvolvimento de 120 e 100 dias, respectivamente, e "BRS Firmeza" (suscetível) e "Dawn" (resistência moderada), com ciclo de desenvolvimento de 120 e 135 dias, respectivamente (TERRES et al., 1999). Foram instalados experimentos a campo, com e sem chance de escolha do hospedeiro, nos anos agrícolas de 1997/98 e 1998/99, na Embrapa Clima Temperado, Pelotas, RS.

$\mathrm{O}$ experimento com chance de escolha consistiu de 10 parcelas, com 96 x 80cm, contendo, cada uma, 48 plantas de arroz das quatro cultivares descritas, instaladas em 10 de dezembro de 1997 e 14 de novembro de 1998. As plantas, em quatro linhas espaçadas a $20 \times 8 \mathrm{~cm}$, foram dispostas em grupos de três, aleatoriamente, no interior da parcela. Aos 25 dias após a emergência das plantas (DAE), gaiolas revestidas com tela de náilon $\left(1,0 \mathrm{~mm}^{2}\right)$ foram colocadas sobre as parcelas. Em seguida, as plantas de cinco parcelas foram infestadas com 48 casais de $\boldsymbol{O}$. oryzae, na densidade de um casal por planta, coletados em lavouras comerciais. Como controle, utilizaram-se cinco parcelas, livres do ataque do inseto.

No mesmo período, foi instalado o experimento sem chance de escolha, sendo utilizadas seis repetições para cada parcela infestada. Como controle, utilizaram-se parcelas livres do ataque do inseto, com seis repetições, totalizando 48 unidades experimentais. Gaiolas revestidas de tela de náilon $\left(1,0 \mathrm{~mm}^{2}\right)$ abrigaram três plantas da mesma cultivar, possibilitando assim o ataque do inseto sem chance de escolha do hospedeiro. Três casais em cópula foram 
utilizados para infestar as plantas de cada parcela, na densidade de um casal por planta. O manejo do solo e as práticas culturais objetivaram simular ao máximo as condições verificadas em lavouras comerciais. A adubação nitrogenada foi realizada em dose única, também a lanço, na diferenciação do primórdio floral, conforme as recomendações técnicas da pesquisa para a cultura do arroz irrigado para a região Sul do Brasil (IRGA, 1995). A irrigação por inundação, realizada no dia que antecedeu as infestações de $\boldsymbol{O}$. oryzae, consistiu em uma lâmina de água uniforme de aproximadamente $15,0 \mathrm{~cm}$, que permaneceu constante até a maturação e a colheita das plantas.

Aos cinco dias após a infestação, correspondendo a $30 \mathrm{DAE}$, em uma e quatro plantas por parcela, nos experimentos sem e com chance de escolha, respectivamente, foram avaliados o número de folhas lesionadas pelos adultos de O. oryzae e o teor de nitrogênio na parte aérea e nas raízes de plantas infestadas e também das livres do ataque do inseto. Aos 25 dias após a infestação, 55 DAE, nos dois anos de experimentação e no mesmo número de plantas citado anteriormente, foram determinados o número e o peso de larvas de $\boldsymbol{O}$. oryzae e o teor de nitrogênio na parte aérea e nas raízes das plantas. Na colheita, seguindo o procedimento descrito para as avaliações anteriores, o teor de nitrogênio foi novamente quantificado e, nos experimentos com chance de escolha, foi determinada a produção de grãos.

O número de larvas por planta foi determinado através da técnica de amostragem adaptada de TUGWELL \& STEPHEN (1981). Após a contagem, as larvas foram pesadas conjuntamente por planta, em balança de precisão. Para as três épocas de determinação do teor de nitrogênio da planta, o sistema radicular foi separado da parte aérea, em laboratório, e lavado em jato de água para remoção do solo aderido às raízes. Em seguida, as duas partes da planta foram mantidas em estufa a $60^{\circ} \mathrm{C}$, até peso constante, e, em seguida, moídas. Para a quantificação do teor de nitrogênio, foi adotada metodologia proposta por TEDESCO et al. (1985), a qual possibilita a recuperação do nutriente de forma semelhante à obtida com os métodos de Kjeldahl. A produção de grãos foi realizada nos experimentos com chance de escolha, sendo a porcentagem de redução de produção de grãos determinada pela diferença entre plantas livres e infestadas pelo inseto. As variáveis avaliadas foram submetidas à análise da variação, conjuntamente para os dois anos de experimentação, sendo as médias comparadas pelo teste de Tukey a 5\% de probabilidade. Correlações lineares entre as variáveis com o teor de nitrogênio em todas as cultivares foram estabelecidas e as significâncias determinadas pelo teste t a $10 \%$ de probabilidade.

\section{RESULTADOS E DISCUSSÃO}

As cultivares de arroz irrigado avaliadas apresentaram diferença na porcentagem de folhas lesionadas e no número de larvas de $\boldsymbol{O}$. oryzae, nas condições de com e sem chance de escolha do hospedeiro (Tabela 1). As cultivares "BR IRGA 410" e "BRS Atalanta" apresentaram maior porcentagem de folhas lesionadas em relação às demais cultivares, nas duas condições. O número de larvas de $\boldsymbol{O}$. oryza por planta foi maior na cultivar "BR IRGA 410", em ambas as condições de experimentação, enquanto que os menores valores foram observados nas cultivares "BRS Firmeza", "Dawn" e "BRS Atalanta", no experimento com chance de escolha, e em "Dawn", no experimento sem chance. Em relação ao peso de larvas, as cultivares foram semelhantes em ambas as condições de avaliação (Tabela 1).

Foram constatadas correlações positivas entre porcentagem de folhas lesionadas, número de larvas do inseto por planta e o teor de nitrogênio da parte aérea da planta, 30 DAE (Tabela 2). Entretanto, o

Tabela 1 - Porcentagem de folhas lesionadas, número e peso de larvas de $\boldsymbol{O}$. oryzae, em cultivares de arroz irrigado. Pelotas, RS, $1997 / 98$ e 1998/99.

\begin{tabular}{lcccc}
\hline Variáveis & \multicolumn{3}{c}{ Cultivares* $^{*}$} \\
\cline { 2 - 5 } & BR IRGA 410 & BRS Firmeza & Dawn & BRS Atalanta \\
\hline Folhas lesionadas $^{1}$ & $36,1 \mathrm{a}$ & $29,4 \mathrm{c}$ & $34,4 \mathrm{~b}$ & $38,3 \mathrm{a}$ \\
Folhas lesionadas $^{2}$ & $34,1 \mathrm{a}$ & $30,9 \mathrm{~b}$ & $31,8 \mathrm{~b}$ & $17,4 \mathrm{~b}$ \\
Número de larvas $^{1}$ & $26,8 \mathrm{a}$ & $15,2 \mathrm{~b} \mathrm{a}$ \\
Número de larvas $^{2}$ & $21,1 \mathrm{a}$ & $10,9 \mathrm{c}$ & $7,9 \mathrm{~d}$ & $18,3 \mathrm{~b}$ \\
Peso de larvas $^{1}$ & $5,0 \mathrm{a}$ & $3,9 \mathrm{a}$ & $3,9 \mathrm{a}$ & $3,9 \mathrm{a}$ \\
Peso de larvas $^{2}$ & $4,2 \mathrm{a}$ & $4,2 \mathrm{a}$ & $3,9 \mathrm{a}$ & $3,9 \mathrm{a}$ \\
\hline
\end{tabular}

Experimentos com $^{1}$ e sem ${ }^{2}$ chance de escolha de hospedeiro.

*Médias seguidas por letras distintas, na mesma linha, diferem entre si pelo teste de Tukey a 5\% de probabilidade.

Ciência Rural, v.36, n.6, nov-dez, 2006. 
Tabela 2 - Coeficientes de correlação linear (r) entre folhas lesionadas, número e peso de larvas de $\boldsymbol{O}$. oryzae com a porcentagem de nitrogênio da parte aérea e das raízes da planta em cultivares de arroz irrigado. Pelotas, RS, 1997/98 e 1998/99.

\begin{tabular}{|c|c|c|c|c|}
\hline \multicolumn{3}{|c|}{ Combinações de Variáveis } & $\begin{array}{c}\text { Com } \\
\text { Chance }\end{array}$ & $\begin{array}{c}\begin{array}{c}\text { Sem } \\
\text { Chance }\end{array} \\
r\end{array}$ \\
\hline Folhas lesionadas ${ }^{1}$ & $\mathrm{x}$ & Nitrogênio & $0,775^{*}$ & $0,946^{*}$ \\
\hline Larvas/planta $^{1}$ & $\mathrm{x}$ & Nitrogênio & $0,925^{*}$ & $0,918^{*}$ \\
\hline Larvas/planta $^{2}$ & $\mathrm{x}$ & Nitrogênio & 0,537 & 0,103 \\
\hline Larvas/planta ${ }^{3}$ & $\mathrm{x}$ & Nitrogênio & 0,092 & 0,189 \\
\hline Peso de larvas ${ }^{3}$ & $\mathrm{x}$ & Nitrogênio & 0,164 & 0,513 \\
\hline
\end{tabular}

${ }^{1}$ parte aérea da planta aos $30 \mathrm{DAE} ;{ }^{2}$ raízes aos $30 \mathrm{DAE} ;{ }^{3}$ raízes aos 55 DAE.

"Significativo pelo teste " $\mathrm{t}$ " a $1 \%$ de probabilidade $(\mathrm{r} \geq 0,917), 5 \%$ $(r \geq 0,811)$ e $10 \%(r \geq 0,729)$.

número e o peso de larvas de $\boldsymbol{O}$. oryzae não mostraram correlações com o teor do nutriente das raízes da planta aos 30 e 55 DAE (Tabela 2).

O nitrogênio é um nutriente que, em teores elevados nas plantas de arroz, aumenta a suscetibilidade destas ao ataque de insetos (BOWLING, 1963; BANG \& TUGWELL, 1976; MENESES \& ELIZALDE, 1980; OLIVEIRA, 1980; MARTINS et al., 1986; STOUT et al., 1998), por tornálas mais atraentes e estimulantes à alimentação pelos insetos. A exemplo do ocorrido com a alimentação de adultos de $\boldsymbol{O}$. oryzae, é freqüente a associação de maiores teores de nitrogênio com a oviposição de gorgulhos-aquáticos (BOWLING, 1963; MENESES \& ELIZALDE, 1980; STOUT et al., 1998). O efeito positivo do nitrogênio sobre a população larval de $\boldsymbol{O}$. oryzae verificado neste trabalho corrobora resultados obtidos com L. oryzophilus (BOWLING, 1963; STOUT et al., 1998) e L. brevirostris (MENESES \& ELIZALDE, 1980).
O teor de nitrogênio, na parte aérea e no sistema radicular, reduziu-se com o desenvolvimento da planta. Na primeira avaliação (30DAE), antes da infestação do inseto, não houve diferença entre plantas da mesma cultivar, livres e infestadas por O oryzae, evidenciando a homogeneidade da área experimental em relação ao nutriente. Entretanto, após o dano das larvas (55 DAE), a redução do teor do nutriente, na parte aérea e nas raízes da planta, foi significativa na maioria das cultivares, com exceção nas raízes de "BRS Firmeza". Na colheita das plantas, a redução do teor de nitrogênio foi significativa, na parte aérea, em todas as cultivares, enquanto que, nas raízes, a significância ficou restrita para "BR IRGA 410" e "BRS Atalanta" com a infestação de $\boldsymbol{O}$. oryzae (Tabela 3).

Houve correlação positiva entre número de larvas e redução do teor do nutriente, na parte aérea das plantas, 55 DAE e na colheita; e, nas raízes, somente 55 DAE (Tabela 4). Com o peso de larvas, a correlação positiva com a redução do teor do nutriente somente ocorreu 55 DAE, na parte aérea da planta (Tabela 1). Tais correlações evidenciam o efeito da população e não do tamanho de larvas na redução do teor de nitrogênio da parte aérea e das raízes das plantas de arroz. Portanto, a ausência de diferença na redução do teor de nitrogênio na cultivar "Dawn" (Tabela 3), além de estar associada a menor número de larvas, provavelmente, também esteja relacionada ao maior ciclo de desenvolvimento da cultivar, o que teria possibilitado maior recuperação do sistema radicular danificado por $\boldsymbol{O}$. oryzae e, conseqüentemente, maior teor do nutriente absorvido. Cultivares de arroz irrigado com maior ciclo de desenvolvimento apresentaram maior capacidade de recuperação do sistema radicular, danificado por larvas de $\boldsymbol{O}$. oryzae, comparadas com as de ciclo curto (CARBONARI et al., 2000), resultando em acréscimo da quantidade de nitrogênio absorvido (CUNHA et al., 2001). A recuperação de raízes, mais

Tabela 3 - Teor de nitrogênio (\%) na parte aérea e nas raízes de cultivares de arroz irrigado, livres (s/i) e infestadas (c/i) por $\boldsymbol{O}$. oryzae, durante o ciclo de desenvolvimento da cultura. Pelotas, RS, 1997/98 e 1998/99.

\begin{tabular}{|c|c|c|c|c|c|c|c|c|c|c|c|c|}
\hline \multirow{3}{*}{ Cultivares } & \multicolumn{6}{|c|}{ Parte aérea } & \multicolumn{6}{|c|}{ Raízes } \\
\hline & \multicolumn{2}{|c|}{$30 \mathrm{DAE}$} & \multicolumn{2}{|c|}{$55 \mathrm{DAE}$} & \multicolumn{2}{|c|}{ Colheita } & \multicolumn{2}{|c|}{$30 \mathrm{DAE}$} & \multicolumn{2}{|c|}{$55 \mathrm{DAE}$} & \multicolumn{2}{|c|}{ Colheita } \\
\hline & $\mathrm{s} / \mathrm{i}$ & $\overline{c / i}$ & $\mathrm{~s} / \mathrm{i}$ & $\overline{c / i}$ & $\mathrm{~s} / \mathrm{i}$ & $\mathrm{c} / \mathrm{i}$ & $\mathrm{s} / \mathrm{i}$ & $\overline{\mathrm{c} / \mathrm{i}}$ & $\mathrm{s} / \mathrm{i}$ & $\overline{c / i}$ & $\mathrm{~s} / \mathrm{i}$ & $\mathrm{c} / \mathrm{i}$ \\
\hline BR IRGA 410 & $3,8 \mathrm{a}$ & $3,8 \mathrm{a}$ & $2,2 \mathrm{a}$ & $1,8 \mathrm{~b}$ & $1,0 \mathrm{a}$ & $0,8 \mathrm{~b}$ & $1,3 \mathrm{a}$ & $1,3 \mathrm{a}$ & $0,9 \mathrm{a}$ & $0,8 \mathrm{~b}$ & $0,6 \mathrm{a}$ & $0,5 \mathrm{~b}$ \\
\hline BRS Firmeza & $3,3 \mathrm{a}$ & $3,3 \mathrm{a}$ & $2,1 \mathrm{a}$ & $1,9 \mathrm{~b}$ & $1,2 \mathrm{a}$ & $1,0 \mathrm{~b}$ & $1,2 \mathrm{a}$ & $1,2 \mathrm{a}$ & $0,9 \mathrm{a}$ & $0,9 \mathrm{a}$ & $0,7 \mathrm{a}$ & $0,7 \mathrm{a}$ \\
\hline Dawn & $3,4 \mathrm{a}$ & $3,4 \mathrm{a}$ & $1,9 \mathrm{a}$ & $1,6 \mathrm{~b}$ & $1,2 \mathrm{a}$ & $1,0 \mathrm{~b}$ & $1,2 \mathrm{a}$ & $1,2 \mathrm{a}$ & $0,9 \mathrm{a}$ & $0,7 \mathrm{~b}$ & $0,6 \mathrm{a}$ & $0,5 \mathrm{a}$ \\
\hline BRS Atalanta & $3,6 \mathrm{a}$ & $3,6 \mathrm{a}$ & $2,1 \mathrm{a}$ & $1,8 \mathrm{~b}$ & $1,2 \mathrm{a}$ & $1,0 \mathrm{~b}$ & $1,2 \mathrm{a}$ & $1,2 \mathrm{a}$ & $1,0 \mathrm{a}$ & $0,8 \mathrm{~b}$ & $0,7 \mathrm{a}$ & $0,6 \mathrm{~b}$ \\
\hline
\end{tabular}

*Médias seguidas por letras distintas, na mesma cultivar, parte de planta e época de avaliação, diferem entre si pelo teste de Tukey a 5\% de probabilidade. 
Tabela 4 - Coeficientes de correlação linear entre número e peso de larvas de $\boldsymbol{O}$. oryzae e redução (sem e com infestação) na porcentagem de nitrogênio da parte aérea de cultivares de arroz irrigado, 55 dias (r) ${ }^{1}$ após a emergência e na colheita $(r)^{2}$ das plantas. Pelotas, RS, $1997 / 98$ e 1998/99.

\begin{tabular}{lllll}
\hline \multicolumn{2}{c}{ Combinações de variáveis } & $(\mathrm{r})^{1}$ & $(\mathrm{r})^{2}$ \\
\hline Número de larvas $^{1}$ & $\mathrm{x}$ & Nitrogênio & $0,965^{*}$ & $0,884^{*}$ \\
Número de larvas $^{2}$ & $\mathrm{x}$ & Nitrogênio & $0,745^{*}$ & 0,697 \\
Peso de larvas $^{1}$ & $\mathrm{x}$ & Nitrogênio & $0,734^{*}$ & 0,623 \\
Peso de larvas $^{2}$ & $\mathrm{x}$ & Nitrogênio & 0,639 & 0,617 \\
Nitrogênio $^{1}$ & $\mathrm{x}$ & Perda de produção & - & $0,903^{*}$ \\
Nitrogênio $^{2}$ & $\mathrm{x}$ & Perda de produção & - & $0,895^{*}$ \\
\hline
\end{tabular}

$\mathrm{N}=$ Número de combinações entre variáveis; ${ }^{1}$ Parte aérea da planta; ${ }^{2}$ Raízes da planta.

"Significativo pelo teste "t" a $1 \%$ de probabilidade $(r \geq 0,917), 5 \%$ $(r \geq 0,811)$ e $10 \%(r \geq 0,729)$.

evidenciada em cultivares de ciclo médio, é atribuída à maior capacidade de regeneração de tecidos danificados, comparativamente a cultivares de ciclo precoce, sendo por isso mais resistentes a $\boldsymbol{O}$. oryzae (MARTINS et al., 2004b).

Com base na associação entre redução percentual do teor de nitrogênio (diferença entre com e sem infestação do inseto), tanto em raízes como na parte aérea, constatou-se que houve uma correlação positiva entre esta variável e a perda de produção (Tabela 4). Assim, em cultivares com menor capacidade de absorção de nitrogênio, sob condições de infestação por $\boldsymbol{O}$. oryzae, é de se esperar a ocorrência de maiores perdas de produtividade.

\section{CONCLUSÕES}

A alimentação de adultos de $\boldsymbol{O}$. oryzae é estimulada pela elevação do teor de nitrogênio da parte aérea das plantas das cultivares de arroz "BR IRGA 410", "BRS Firmeza", "Dawn" e "BRS Atalanta". Apenas a cultivar "Dawn", que hospedou menor quantidade de larvas de $\boldsymbol{O}$. oryzae, apresentou maior ciclo de desenvolvimento fenológico e recuperou o teor de nitrogênio após o dano do inseto. Plantas das quatro cultivares avaliadas, sob ataque de larvas do inseto, apresentam redução do teor de nitrogênio e, por conseguinte, perda na produção de grãos.

\section{REFERÊNCIAS}

BANG, Y.H.; TUGWELL, P. Adult rice water weevil feeding preferences for rice plants and leaves of different ages. Arkansas: Agricultural Experimental Station, 1976. 12p. (Report Series, 231).
BOWLING, C.C. Effect of nitrogen levels on rice water weevil populations. Journal of Economic Entomology, v.55, p.826$827,1963$.

BOWLING, C.C. Breeding for host plant resistance to rice field insects in the U.S.A. In: HARRIS, M.K. Biology and breeding for resistance to arthropods and pathogens in agricultural plants. College Station: Texas A \& M University, 1980. p.329-364.

CARBONARI, J.J. et al. Relação entre flutuação populacional de Oryzophagus oryzae (Costa Lima) (Coleoptera: Curculionidae) e período de perfilhamento de cultivares de arroz irrigado. Anais da Sociedade Entomológica do Brasil, v.29, p.361-366, 2000.

CUNHA, U.S. et al. Recuperação de plantas de arroz irrigado danificadas por larvas de Oryzophagus oryzae (Costa Lima, 1936) (Coleoptera: Curculionidae) pela adubação nitrogenada em cobertura. Revista Brasileira de Agrociência, v.7, n.1, p.58-63, 2001.

FERREIRA, E.; MARTINS, J.F.S. Insetos prejudiciais ao arroz no Brasil e seu controle. Goiânia: EMBRAPA, CNPAF, 1984. 67p. (Documentos, 11).

INSTITUTO RIOGRANDENSE DO ARROZ. Recomendações técnicas da pesquisa para o Sul do Brasil. Cachoeirinha: 1995. 83p.

MARTINS, J.F.S. Problemática da bicheira-da-raiz no Rio Grande do Sul. In: REUNIÃO NACIONAL DE PESQUISA DE ARROZ, 4., 1990, Goiânia, GO. Resumos... Goiânia: EMBRAPA, CNPAF, 1990. p.29.

MARTINS, J.F.S.; BOTTON, M. Controle de insetos da cultura do arroz. In: PESKE, S.T. et al. Produção de arroz irrigado. Pelotas: UFPel, 1996. p.273-299.

MARTINS, J.F.S.; FERREIRA, E. Caracterização e controle da bicheira-da-raiz do arroz. Goiânia: EMBRAPA, CNPAF. 1980. 14p. (Circular Técnica, 9).

MARTINS, J.F.S.; TERRES, A.L.S. Avaliação de germoplasma de arroz visando resistência a Oryzophagus oryzae (Costa Lima). Anais da Sociedade Entomológica do Brasil, v.24, p.445453, 1995.

MARTINS, J.F.S. et al. Adubação nitrogenada e controle da bicheira da raiz do arroz. Lavoura Arrozeira, v.40, p.8-11, 1986.

MARTINS, J.F.S. et al. Controle de Oryzophagus oryzae (Costa Lima) através da pulverização foliar com inseticidas piretróides. Anais da Sociedade Entomológica do Brasil, v.25, p.217221, 1996.

MARTINS, J.F.S et al. Descrição e manejo integrado de insetospraga em arroz irrigado. In: GOMES, A. da S.; MAGALHÃES JUNIOR, A.M. de. Arroz irrigado no Sul do Brasil. Brasilia, DF: Embrapa Informação Tecnológica, 2004a. Cap.19, p.635675 .

MARTINS, J.F.S. et al. Simulação do dano causado por larvas de Oryzophagus oryzae a cultivares de arroz irrigado. Ciência Rural, v.34, n.3, p.653-659, 2004 b. 
MENESES, R.; ELIZALDE, R. Influencia de la fertilización mineral en el cultivo del arroz sobre la población de Lissorhoptrus brevirostris (Coleoptera: Curculionidade). Ciência Técnica Agrícola, v.3, p.49-69, 1980.

N'GUESSAN, F.K.N. et al. Evaluation of rice tissue culture lines for resistance to the rice water weevil (Coleoptera: Curculionidae). Journal of Economic Entomology, v.87, p.504-513, 1994.

OLIVEIRA, J.V. de. Estudo de níveis de uréia em bicheira da raiz no arroz irrigado. In: REUNIÃO DA CULTURA DO ARROZ IRRIGADO, 10., 1980, Porto Alegre, RS. Anais... Porto Alegre: IRGA, 1980. p.211-212.

SMITH, C.M.; ROBINSON, J.F. Evaluation of rice cultivars grown in North America for resistance to the rice water weevil. Environmental Entomology, v.11, p.334-336, 1982.

STOUT, M.J. et al. Impact of fertilization rate on rice resistance and tolerance to the rice water weevil: a preliminary report. In: RICE RESEARCH STATION. AGRICULTURAL CENTER. Annual research report 90 th. Crowley: Louisiana State University, 1998. p.496-499.

TEDESCO, M.J. et al. Análise de solo, plantas e outros materiais. Porto Alegre: UFRGS, 1985. 118p. (Boletin Técnico de Solos, 5).

TERRES, A.L.S. et al. Melhoramento genético de arroz irrigado na Embrapa Clima Temperado: 10. BRS Firmeza e BRS Atalanta, novas cultivares para a orizicultura gaúcha. In: CONGRESSO BRASILEIRO DE ARROZ IRRIGADO, 1.; REUNIÃO DA CULTURA DO ARROZ IRRIGADO, 23., 1999, Pelotas, RS. Anais... Pelotas: Embrapa Clima Temperado, 1999. p.158161.

TUGWELL, N.P.; STEPHEN, F.M. Rice water weevil seasonal abundance, economic levels, and sequential sampling plants. Fayetteville: Agricultural Experiment Station, 1981. 16p. (Bulletin, 849) 